\title{
Correção de estrabismo paralítico por injeção de toxina botulínica
}

\author{
Management of paralytic strabismus by injection of botulinum toxin
}

Raquel Wattiez ${ }^{(1)}$

Fábio Henrique Cacho Casanova ${ }^{(2)}$

Rosana N. Pires da Cunha ${ }^{(3)}$

Tomás Scalamandré Mendonça ${ }^{(4)}$

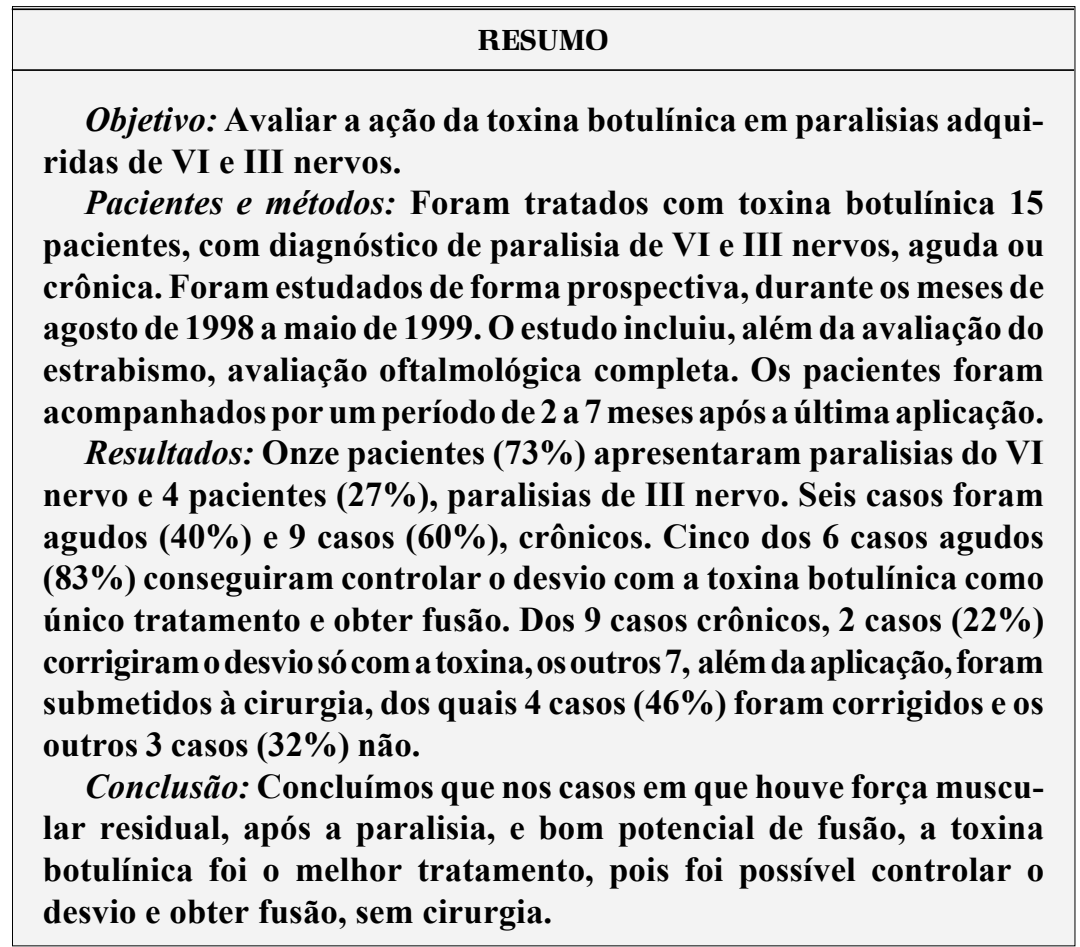

Palavras-chave: Toxina botulínica; Estrabismo paralítico adquirido.

\section{INTRODUÇÃ̃O}

A toxina botulínica, produzida pelo Clostridium botulinum, causa bloqueio das conexões colinérgicas da placa neuromotora por inibição da acetilcolina, denervando funcionalmente o músculo. Quando é injetada no músculo, produz enfraquecimento ou paralisia temporária dose-dependente ${ }^{1}$.

A idéia de injetar um agente farmacológico dentro da musculatura ocular extrínseca no ser humano, para produzir debilitamento prolongado ou permanente, foi concebida por Conrad Behrens ${ }^{2}$. Ele usou álcool, que se mostrou ineficaz. Adotando a idéia de Behrens, Allan Scott et al experimentaram várias drogas, tais como, isopropil-fluorofosfato (DFP), neurotoxina Bungarus (veneno de cobra), álcool e toxina botulínica tipo A, com o intuito de paralisar os músculos extraoculares do macaco Rhesus ${ }^{2-4}$.

Scott observou as propriedades desta toxina e concluiu que a toxina botulínica tipo A seria a droga ideal para produzir uma paralisia transitória na musculatura ocular extrínseca, e também alterações permanentes no alinhamento ocular sem sérios efeitos colaterais ${ }^{4-6}$. 
A partir de 1970, o FDA (Food and Drug Administration) liberou o uso da medicação, em caráter experimental, para um grupo de pesquisadores em todo o mundo orientados pelo próprio Allan $\operatorname{Scott}^{7}$. Suas observações preliminares mostraram que a toxina botulínica pode ser usada como alternativa ao tratamento cirúrgico convencional (recuo-ressecção ou transposição de músculos, dependendo do tipo de paralisia) ${ }^{5,6}$.

Posteriormente, o uso da toxina foi ampliado, sendo utilizada no tratamento de várias doenças neuromusculares, tais como blefaroespasmo, espasmo hemifacial, mioquimia, entrópio, oftalmopatia da moléstia de Graves, nistagmo, regeneração aberrante de III nervo ${ }^{5,6}$.

Em dezembro de 1989, a toxina botulínica foi liberada para uso terapêutico em blefaroespasmo e estrabismo, em pacientes com idade superior a 12 anos ${ }^{5}$.

A toxina botulínica no tratamento do estrabismo parece produzir efeitos não tão previsíveis e estáveis como a cirurgia convencional, mas pode ser efetiva em certos tipos específicos de estrabismo, como desvios de pequenos ângulos, desvios secundários (sensoriais), paralisias agudas do VI nervo, oftalmopatia subaguda da moléstia de Graves, hipo e hipercorreções póscirúrgicas, estrabismos pós-cirurgia de descolamento de retina e pacientes sem condições clínicas para anestesia geral ou para correção cirúrgica ${ }^{5,8}$.

O procedimento cirúrgico é ainda o método mais apropriado nos casos de grandes desvios, quando há muitos músculos envolvidos, em estrabismos com incomitâncias alfabéticas associadas, ou ainda quando houver cicatrizes de tecido perimuscular.

O mecanismo de ação da droga ocorre no nível das terminações nervosas, bloqueando a liberação de Acetilcolina (Ach) nas sinapses, em três etapas: ligação, internalização e paralisia. A paralisia do músculo é causada pela inibição da liberação de Ach, não interferindo, portanto, na propagação do impulso nervoso, nem na síntese ou no armazenamento da Ach. A hipótese mais provável é que a toxina una-se às vesículas que contêm Ach, impedindo a exocitose cálciodependente ${ }^{9}$.

O efeito paralítico da toxina é dose-dependente e o pico de ação ocorre entre o $5^{\circ}$ e o $7^{\circ}$ dia após injeção.

O efeito pode persistir por 3 a 6 meses após a aplicação. Depois da recuperação da paralisia química causada pela toxina, o músculo injetado pode ou não recuperar o nível funcional pré-injeção ${ }^{6-8}$.

Qualquer paralisia de média a longa duração de um músculo ocular extrínseco sempre causa contratura (encurtamento) do seu antagonista, a qual impede a recuperação total do paciente, mesmo que não exista mais a paralisia inicial ${ }^{10}$.

Existem muitos estudos sobre a toxina botulínica em diferentes tipos de estrabismos e com ótimos resultados, sobretudo em paralisias agudas de VI nervo. Esses estudos também mostram que, em paralisias crônicas, a toxina pode desfazer a contratura adquirida do antagonista, diminuindo assim o número de músculos a serem operados ${ }^{10}$.
Trabalhos mais recentes mostram que o uso em crianças pode resultar num método alternativo e eficiente ${ }^{11}$.

\section{OBJETIVO}

O objetivo deste estudo foi avaliar a ação da toxina botulínica em pacientes portadores de estrabismos paralíticos adquiridos (paralisia de III e VI nervos).

\section{PACIENTES E MÉTODOS}

Foram estudados prospectivamente 15 pacientes atendidos no Setor de Estrabismo do Departamento de Oftalmologia da UNIFESP - EPM, com diagnóstico de paralisia adquirida do III e VI nervos cranianos de caráter crônico ou agudo, entre os meses de agosto de 1998 a maio de 1999. Não foram estudados pacientes com paralisias congênitas.

A acuidade visual (AV) mínima para incluir os pacientes foi de 20/200 no pior olho, com diferença máxima de quatro linhas entre ambos os olhos, quando existia ambliopia.

O desvio ocular foi determinado pelo teste de "prisma e cover" alternado ou pelo teste de Krimsky. As funções dos músculos comprometidos (agonista e antagonista) foram avaliadas pelas ducções oculares graduadas de -6 (limitação máxima) a 0 (ducção normal dentro do campo de ação muscular).

Para diferenciar se o paciente apresentava paresia ou paralisia foi realizado o teste de forças geradas. O grau de contratura do antagonista foi determinado pelo teste de ducção forçada.

A toxina botulínica tipo A utilizada no estudo foi do laboratório Allergan $\left(\right.$ Botox $\left.^{\circledR}\right)$. A droga foi injetada no músculo extraocular, usando uma agulha hipodérmica descartável $37 \mathrm{~mm} \times 27 \mathrm{G}$, que possui um eletrodo monopolar adaptado a um eletromiógrafo com sinal sonoro, construído especificamente para esse fim (marca Allergan), para assegurar a colocação exata da agulha dentro do músculo, próxima da placa neuromotora muscular. Foi utilizada para tal procedimento anestesia tópica (cloridrato de proximetacaína $0,5 \%$ ). A dose de toxina injetada por músculo foi calculada de acordo com a magnitude do desvio ocular, como no seguinte esquema:

\section{Para desvio horizontal}

$$
\begin{aligned}
& <25^{\Delta} \Rightarrow 2,5 \mathrm{U}^{*} \\
& 25^{\Delta}-50^{\Delta} \Rightarrow 5 \mathrm{U} \\
& >50^{\Delta} \Rightarrow 7,5 \text { a } 10 \mathrm{U}
\end{aligned}
$$

\section{Para desvio vertical}

$15^{\Delta}-20^{\Delta} \Rightarrow 5$ a $7,5 \mathrm{U}$

Desvios menores não foram considerados para tratamento

Os pacientes foram observados mediante um protocolo específico, onde se incluiu avaliação oftalmológica completa, além de exame da motilidade extrínseca.

\footnotetext{
* Unidade (dose letal no rato, LD 50)
} 
O tempo de seguimento mínimo foi de 2 meses e o máximo de 8 meses, após realizado o último procedimento.

Em alguns casos crônicos, além do tratamento com a toxina botulínica foi associado procedimento cirúrgico. Novas aplicações foram realizadas nos casos em que o desvio não foi modificado com a primeira injeção, a fim de tentar diminuir a contratura do antagonista e posteriormente associar procedimento cirúrgico.

\section{RESULTADOS}

Dos 15 pacientes incluídos, 7 eram do sexo masculino e 8 do sexo feminino. A idade dos pacientes variou de 9 a 68 anos (média $=43,6$ anos).

Os fatores etiológicos das paralisias nestes casos foram: trauma crânio-encefálico, 8 casos $(53,3 \%)$; tumor intracraniano, 1 caso (6,6\%); aneurisma cerebral, 3 casos $(20 \%)$; afecção do SNC por HIV(+), 1 caso $(6,6 \%)$ e $2(13 \%)$ casos de etiologia desconhecida (Tabela 1).

Onze pacientes (73\%) apresentaram paralisia do VI nervo, sendo que um deles bilateral. Quatro pacientes (36\%) apresentaram paralisia do III nervo incompleta, dois casos apresentando acometimento pupilar. Seis pacientes (40\%) foram classificados como paralisias agudas (até 6 meses de evolução). Nove pacientes (60\%), como paralisia crônica (mais de 6 meses de evolução).

\section{- VI Nervo}

Onze pacientes ( $73 \%$ ) com paralisia do VI nervo apresentaram desvio pré-injeção que variou entre ET de 20 dioptrias $\left(20^{\Delta}\right)$ e ET $75^{\Delta}$ (média $43,1^{\Delta}$ ).

Nas primeiras duas semanas após aplicação, houve grande variação na resposta observada. Os casos 2, 5, 12 e 14 apre- sentaram regressão completa do desvio horizontal. Nos casos $7,8,9,11$ e 15 houve diminuição do desvio horizontal superior a $15^{\Delta}$, não havendo boa resposta apenas no caso 3. Foi observada inversão do desvio no caso 6 , cujo paciente apresentava ET $40^{\Delta}$, tendo resultado numa XT de $35^{\Delta}$ após aplicação (amplitude de variação do desvio, $75^{\Delta}$ ).

Todos os casos foram acompanhados entre 2 e 6 meses, exceto o caso 3. Neste caso em particular, foram feitas duas aplicações de toxina botulínica com intervalo de 2 semanas entre elas. Após a segunda aplicação, foi indicada cirurgia devido à falta de resposta ao medicamento (Tabela 2).

Em $80 \%$ dos casos agudos, observou-se correção completa do desvio.

Sessenta e sete por cento dos casos crônicos obtiveram correção do desvio de mais de $50 \%$ entre os 2 meses que foram acompanhados. Nestes casos, a toxina botulínica foi utilizada com finalidade de diminuir a contratura do músculo antagonista havendo necessidade de associar procedimento cirúrgico para correção do desvio ainda na vigência de ação da droga (Tabela 2).

\section{- III Nervo}

Os casos 1, 4, 10 e 13 apresentaram paralisia do III nervo com desvio pré injeção variando de XT $25^{\Delta}$ a XT $50^{\Delta}$ e HT (hipertropia) de $10^{\Delta}$ a HT $20^{\Delta}$ (média XT $35^{\Delta}$ e HT $13^{\Delta}$ ). Duas semanas após a aplicação, foi observada diminuição do desvio horizontal de aproximadamente $20^{\Delta} \mathrm{em}$ todos os pacientes. Nos casos 4 e 10 houve resolução completa da exotropia, não havendo resposta em relação à hipertropia, no caso 4 . Em 75\% dos casos foi observada correção completa do desvio até o último seguimento realizado (Tabela 3).

Estes pacientes foram acompanhados ambulatorialmente por no mínimo dois meses. Na maioria dos casos, houve a necessidade de associar procedimento cirúrgico com o intuito de alcançar o melhor alinhamento ocular.

\begin{tabular}{|c|c|c|c|c|}
\hline $\mathbf{N}^{\circ}$ Caso & Agudo/crônico & Etiologia & $\begin{array}{c}\text { Tempo evolução } \\
\text { da afecção }\end{array}$ & Diagnóstico \\
\hline 1 & Crônico & Idiopática & 5 anos & Paralisia III nervo D Incompleta \\
\hline 2 & Crônico & TCE & 1 ano & Paralisia VI nervo E \\
\hline 3 & Crônico & Aneurisma cerebral & 2 anos & Paralisia VI nervo D \\
\hline 4 & Crônico & HIV (+) / TBC Toxoplasmose SNC & 1 ano & Paresia III nervo incompleta $E$ + acometimento pupilar \\
\hline 5 & Agudo & TCE & 4 meses & Paralisia VI Nervo E \\
\hline 6 & Agudo & TCE & 6 meses & Paresia VI Nervo E \\
\hline 7 & Crônico & TCE & 1 ano & Paralisia VI Nervo E \\
\hline 8 & Crônico & TU & 5 anos & Paralisia VI Nervo E \\
\hline 9 & Crônico & Aneurisma cerebral & 2 anos & Paralisia VI Nervo D \\
\hline 10 & Crônico & Aneurisma cerebral & 2 anos & Paralisia III nervo E + Sd Parinaud + Paralisia IV nervo \\
\hline 11 & Crônico & TCE & 2 anos & Paralisia VI Nervo D \\
\hline 12 & Agudo & Idiopática & 2 meses & Paralisia bilateral VI nervo \\
\hline 13 & Agudo & TCE & $11 / 2$ meses & Paresia III nervo incompleta $\mathrm{E}+$ acometimento pupilar \\
\hline 14 & Agudo & TCE & 2 meses & Paralisia VI Nervo E \\
\hline 15 & Agudo & TCE & 2 meses & Paralisia VI Nervo E \\
\hline
\end{tabular}




\begin{tabular}{|c|c|c|c|c|c|c|c|c|}
\hline $\begin{array}{l}\mathrm{N}^{\circ} \\
\text { caso }\end{array}$ & Sexo & Idade & Músculo injetado & $\begin{array}{c}\text { Pré-injeção } \\
\text { Desvio (em PPO) }\end{array}$ & $\begin{array}{l}2 \text { semanas } \\
\text { Desvio }\end{array}$ & $\begin{array}{l}\text { Seguime } \\
2 \text { meses } \\
\text { Desvio }\end{array}$ & $\begin{array}{c}\text { ós-injeção } \\
4 \text { meses } \\
\text { Desvio }\end{array}$ & $\begin{array}{c}6 \text { meses } \\
\text { Desvio }\end{array}$ \\
\hline 2 & M & 34 & $\mathrm{RM}-\mathrm{OD}$ & ET $50^{\Delta}$ & $\begin{array}{l}\text { HTD } 6^{\Delta} \\
\text { HTD } 8^{\Delta}\end{array}$ & ET $6^{\Delta}$ & -- & -- \\
\hline $3^{*}$ & $\mathrm{~F}$ & 55 & $\begin{array}{l}\text { RM - OD } \\
\text { HoTD } 10^{\Delta}\end{array}$ & $\begin{array}{c}\text { ET } 75^{\Delta} \\
\text { HoTD } 10^{\Delta}\end{array}$ & ET $70^{\Delta}$ & -- & -- & -- \\
\hline $3^{* *}$ & & & $\begin{array}{l}\text { RM - OD } \\
\text { HoT } 10^{\Delta}\end{array}$ & $\begin{array}{l}\text { ET } 75^{\Delta} \\
\text { HoTD } 10^{\Delta}\end{array}$ & ET $75^{\Delta}$ & -- & -- & -- \\
\hline $5^{*}$ & $\mathrm{~F}$ & 58 & $\mathrm{RM}-\mathrm{OE}$ & ET $40^{\Delta}$ & $\begin{array}{l}\text { HTE } 8^{\Delta} \\
\text { HTE } 15^{\Delta}\end{array}$ & ET $25^{\Delta}$ & -- & -- \\
\hline $5^{\star \star}$ & & & $\begin{array}{l}\text { RM - OE } \\
\text { HTE } 15^{\Delta}\end{array}$ & $\begin{array}{l}\text { ET 25 } \\
\text { HTE } 12^{\Delta}\end{array}$ & $\begin{array}{l}\text { ET 20 } \\
\text { HTE } 15^{\Delta}\end{array}$ & $\begin{array}{l}\text { ET } 20^{\Delta} \\
\text { HTE } 12^{\Delta}\end{array}$ & $\mathrm{T} 20^{\Delta}$ & -- \\
\hline 6 & $\mathrm{~F}$ & 20 & $\mathrm{RM}-\mathrm{OE}$ & ET $40^{\Delta}$ & $\mathrm{XT} 35^{\Delta}$ & XT $20^{\Delta}$ & ORTO & ORTO \\
\hline 7 & M & 62 & $\mathrm{RM}-\mathrm{OE}$ & ET $50^{\Delta}$ & ET $25^{\Delta}$ & ET $25^{\Delta}$ & -- & - \\
\hline $8^{*}$ & $\mathrm{~F}$ & 09 & $\begin{array}{l}\mathrm{RM}-\mathrm{OE} \\
\mathrm{HTE} 8^{\Delta}\end{array}$ & ET $40^{\Delta}$ & $\begin{array}{l}\text { ET 25 } \\
\text { HTE } 4^{\Delta}\end{array}$ & ET $35^{\Delta}$ & -- & -- \\
\hline $8^{* *}$ & & & $\begin{array}{l}\text { RM - OE } \\
\text { HTE } 4^{\Delta}\end{array}$ & $\begin{array}{l}\text { ET } 35^{\Delta} \\
\text { HTE } 12^{\Delta}\end{array}$ & $\begin{array}{l}\text { ET } 5^{\Delta} \\
\text { HTE } 4^{\Delta}\end{array}$ & ET $20^{\Delta}$ & -- & -- \\
\hline 9 & $\mathrm{~F}$ & 42 & $\mathrm{RM}-\mathrm{OD}$ & $\begin{array}{l}\text { ET } 35^{\Delta} \\
\text { HTD } 6^{\Delta}\end{array}$ & $\begin{array}{c}\text { ET } 8^{\Delta} \\
\text { HTD } 4^{\Delta}\end{array}$ & ET $12^{\Delta}$ & -- & -- \\
\hline 11 & M & 14 & $\begin{array}{l}\text { RM - OD } \\
\text { HTD } 6^{\Delta}\end{array}$ & ET $45^{\Delta}$ & ET $25^{\Delta}$ & ET $25^{\Delta}$ & -- & -- \\
\hline 12 & $M$ & 68 & $\mathrm{RM}-\mathrm{OE}$ & ET $20^{\Delta}$ & ORTO & ORTO & ORTO & ORTO \\
\hline 14 & $M$ & 67 & $\mathrm{RM}-\mathrm{OE}$ & ET $50^{\Delta}$ & HTE $8^{\Delta}$ & HTE $8^{\Delta}$ & ORTO & Não compareceu \\
\hline 15 & $M$ & 60 & $\mathrm{RM}-\mathrm{OE}$ & ET $30^{\Delta}$ & ET $8^{\Delta}$ & ET $8^{\Delta}$ & ORTO & Não compareceu \\
\hline
\end{tabular}

\begin{tabular}{|c|c|c|c|c|c|c|c|c|}
\hline \multirow[b]{2}{*}{$\begin{array}{l}\mathrm{N}^{\circ} \\
\text { caso }\end{array}$} & \multirow[b]{2}{*}{ Sexo } & \multirow[b]{2}{*}{ Idade } & \multirow[b]{2}{*}{$\begin{array}{l}\text { Músculo } \\
\text { injetado }\end{array}$} & \multirow[b]{2}{*}{$\begin{array}{c}\text { Pré-injeção } \\
\text { Desvio (em PPO) }\end{array}$} & \multicolumn{4}{|c|}{ Seguimento pós-injeção } \\
\hline & & & & & $\begin{array}{l}2 \text { semanas } \\
\text { Desvio }\end{array}$ & $\begin{array}{l}2 \text { meses } \\
\text { Desvio }\end{array}$ & $\begin{array}{c}4 \text { meses } \\
\text { Desvio }\end{array}$ & $\begin{array}{c}6 \text { meses } \\
\text { Desvio }\end{array}$ \\
\hline \multirow[t]{2}{*}{1} & $\mathrm{~F}$ & 19 & $\mathrm{RL}-\mathrm{OD}$ & XT $50^{4}$ & XT $30^{4}$ & $\mathrm{XT} 30^{4}$ & -- & -- \\
\hline & & & $\mathrm{RS}-\mathrm{OD}$ & HTD $20^{\Delta}$ & HTD $10^{\Delta}$ & HTD $10^{\Delta}$ & & \\
\hline \multirow[t]{2}{*}{4} & $\mathrm{~F}$ & 30 & $\mathrm{RL}-\mathrm{OD}$ & $\mathrm{XT} 25^{\Delta}$ & ET $10^{\Delta}$ & ET $15^{\Delta}$ & $\times 6^{\Delta}$ & $\times 8^{\Delta}$ \\
\hline & & & $\mathrm{RL}-\mathrm{OE}$ & HTD $10^{\Delta}$ & HTD $8^{\Delta}$ & HTD $8^{\Delta}$ & & \\
\hline \multirow[t]{2}{*}{$10^{*}$} & $\mathrm{~F}$ & 52 & $\mathrm{RL}-\mathrm{OE}$ & $\mathrm{XT} 25^{\Delta}$ & ORTO & $\mathrm{XT} 20^{\Delta}$ & XT $20^{\Delta}$ & \\
\hline & & & $\mathrm{RS}-\mathrm{OE}$ & HTE $15^{\Delta}$ & & HTE $10^{\Delta}$ & HTE $10^{\Delta}$ & \\
\hline \multirow[t]{2}{*}{$10^{* *}$} & & & $\mathrm{RL}-\mathrm{OE}$ & XT $20^{\Delta}$ & $\times 6^{4}$ & $\mathrm{X} 12^{\Delta}$ & $X 15^{\Delta}$ & $\times 15^{\Delta}$ \\
\hline & & & & HTE $10^{\Delta}$ & HTE $2^{\Delta}$ & HTE $4^{\Delta}$ & HTE $2^{\Delta}$ & $H(T) 2^{\Delta}$ \\
\hline \multirow[t]{2}{*}{13} & M & 65 & $\mathrm{RL}-\mathrm{OE}$ & $\mathrm{XT} 40^{\Delta}$ & XT 20 $0^{\Delta}$ & ET $10^{\Delta}$ & $E 2^{\Delta}$ & ORTO \\
\hline & & & & HTE $10^{\Delta}$ & & & & \\
\hline
\end{tabular}

Dentre os 15 pacientes incluídos no estudo, 8 foram submetidos à cirurgia, sendo apenas um desses casos considerado como agudo (caso 5). Houve resposta satisfatória em 5 pacientes (62\%), variando o resultado de ortotropia a $10^{\Delta}$ de desvio (tabela 4).

Dos 6 pacientes que apresentaram paralisias agudas, 5 (casos $6,12,13,14,15)$ apresentaram ortotropia $(83 \%)$, avaliados 4 a 6 meses após aplicação. Um único paciente necessitou de duas aplicações e cirurgia (caso 5) (Tabela 5).
Dos casos com paralisias crônicas, os casos 4 e 10 não precisaram de cirurgia após a aplicação (22\%), alcançando alinhamento ocular e fusão em PPO (posição primária do olhar), permanecendo assim até o último exame realizado (seguimento de 6 meses).

Baseado nos critérios adotados na metodologia do trabalho, realizamos nova aplicação de toxina botulínica nos casos $3,5,8$ e 10 devido à ausência de resposta ou recorrência do 


\begin{tabular}{|c|c|c|c|c|c|c|}
\hline № caso & $\begin{array}{c}\text { Desvio } \\
\text { Pré-injeção }\end{array}$ & $\begin{array}{l}\text { Seguimento } \\
\text { após injeção }\end{array}$ & $\begin{array}{c}\text { Desvio } \\
\text { Pós-injeção }\end{array}$ & $\begin{array}{l}\text { Procedimento } \\
\text { Cirúrgico }\end{array}$ & $\begin{array}{l}\text { Seguimento } \\
\text { após cirurgia }\end{array}$ & $\begin{array}{c}\text { Desvio } \\
\text { Final }\end{array}$ \\
\hline 1 & $\begin{array}{c}\text { XT } 50^{\Delta} \\
\text { HTD } 20^{\Delta}\end{array}$ & 2 meses & $\begin{array}{c}\text { XT } 30^{\Delta} \\
\text { HTD } 10^{\Delta} \\
\text { ET } 6^{\Delta}\end{array}$ & $\begin{array}{l}\text { Rs - RI-OD } \\
\text { Rs - RM-OD }\end{array}$ & 7 meses & $\begin{array}{l}\text { XT } 10^{\Delta} \\
\text { HTD } 4^{\Delta}\end{array}$ \\
\hline 2 & $\begin{array}{l}\text { ET } 50^{\Delta} \\
\text { ET } 75^{\Delta}\end{array}$ & 2 meses & $\begin{array}{l}\text { HTD } 10^{\Delta} \\
\text { ET } 70^{\Delta}\end{array}$ & Rs-RL-OD & 6 meses & ORTO \\
\hline $3^{*}$ & $\begin{array}{l}\text { HoTD } 10^{\Delta} \\
\text { ET } 75^{\Delta}\end{array}$ & 2 semanas & $\begin{array}{l}\text { HoTD10 } \\
\text { ET } 75^{\Delta}\end{array}$ & Rs $-\bar{R}$ R-OD & - & HoTD $20^{\Delta}$ \\
\hline $3^{* *}$ & НоТ $10^{\Delta}$ & 2 semanas & $\begin{array}{l}\text { HoTD } 10^{\Delta} \\
\text { ET } 25^{\Delta}\end{array}$ & $\mathrm{Rc}-\mathrm{RM}-\mathrm{OD}$ & 8 meses & ET $10^{\Delta}$ \\
\hline $5^{*}$ & $\begin{array}{l}\text { ET } 40^{\Delta} \\
\text { ET } 25^{\Delta}\end{array}$ & 2 meses & $\begin{array}{l}\text { HTE } 6^{\Delta} \\
\text { ET } 20^{\Delta}\end{array}$ & - & - & - \\
\hline $5^{\star *}$ & HTE $6^{\Delta}$ & 4 meses & HTE $12^{\Delta}$ & $\begin{array}{l}\text { Rs - RL-OE } \\
\text { Rs - RL-OE }\end{array}$ & 4 meses & HTE $10^{\Delta}$ \\
\hline 7 & $\begin{array}{l}\text { ET } 50^{\Delta} \\
\text { ET } 40^{\Delta}\end{array}$ & $\begin{array}{l}2 \text { meses } \\
2 \text { meses }\end{array}$ & $\begin{array}{l}\text { ET } 25^{\Delta} \\
\text { ET } 35^{\Delta}\end{array}$ & Transposição muscular & 4 meses & $\begin{array}{l}\text { ET } 4^{\Delta} \\
\text { HoT } 12^{\Delta}\end{array}$ \\
\hline $8^{*}$ & $\begin{array}{l}\text { HTE } 8^{\Delta} \\
\text { ET } 35^{\Delta}\end{array}$ & & $\begin{array}{l}\text { HTE } 4^{\Delta} \\
\text { ET } 20^{\Delta}\end{array}$ & Transposição & - & - \\
\hline $8^{* *}$ & HTE $4^{\Delta}$ & 2 meses & $\begin{array}{l}\text { HTE } 4^{\Delta} \\
\text { ET } 12^{\Delta}\end{array}$ & $+\mathrm{Rc}-\mathrm{OI}-\mathrm{OE}$ & 4 meses & $\begin{array}{c}\text { ET } 4^{\Delta} \\
\text { HoTE } 8^{\Delta}\end{array}$ \\
\hline 9 & $\begin{array}{l}\text { ET } 35^{\Delta} \\
\text { ET } 45^{\Delta}\end{array}$ & 2 meses & HTD $4^{\Delta}$ & Rs - RL-OD & 8 meses & ORTO \\
\hline 11 & HTD $6^{\Delta}$ & 2 meses & ET $25^{\Delta}$ & $\begin{array}{l}\text { Rs - RL-OD } \\
\text { Rc - RM-OE }\end{array}$ & $\begin{array}{l}6 \text { meses } \\
2 \text { meses }\end{array}$ & $\begin{array}{l}\text { ET } 20^{\Delta} \\
\text { ET } 10^{\Delta}\end{array}$ \\
\hline
\end{tabular}

\begin{tabular}{|c|c|c|c|c|c|c|}
\hline $\begin{array}{l}\mathrm{N}^{\circ} \\
\text { caso }\end{array}$ & $\begin{array}{c}\text { Tempo de Evolução } \\
\text { da Paralisia }\end{array}$ & Músculo Injetado & № Aplicações & Desvio Pré-injeção & Seguimento & Desvio Final \\
\hline 5 & 4 meses & $\mathrm{RM}-\mathrm{OE}$ & 2 & ET $40^{\Delta}$ & 4 meses & ET $20^{\Delta}$ HTE $12^{\Delta}$ \\
\hline 6 & 6 meses & $\mathrm{RM}-\mathrm{OE}$ & 1 & ET $40^{\Delta}$ & 6 meses & ORTO \\
\hline 12 & 2 meses & $\mathrm{RM}-\mathrm{OE}$ & 1 & ET $20^{\Delta}$ & 6 meses & ORTO \\
\hline $13^{*}$ & 2 meses & $\mathrm{RL}-\mathrm{OE}$ & 1 & XT $40^{\Delta}$ HTE $10^{\Delta}$ & 6 meses & $E 2^{\Delta}$ \\
\hline 14 & 2 meses & $\mathrm{RM}-\mathrm{OE}$ & 1 & ET $50^{\Delta}$ & 4 meses & ORTO \\
\hline 15 & 2 meses & $\mathrm{RM}-\mathrm{OE}$ & 1 & ET $30^{\Delta}$ & 4 meses & ORTO \\
\hline
\end{tabular}

desvio. No caso 3, houve necessidade de associar cirurgia após segunda aplicação. Durante o procedimento cirúrgico, foi observada intensa fibrose circunjacente ao músculo reto medial. Após ressecção do tecido fibroso, constatou-se normalização do teste de ducções passivas podendo explicar o insucesso da toxina previamente aplicada.

Foram observados alguns efeitos colaterais após a aplicação da toxina botulínica, sendo ptose palpebral temporária (9 casos, $60 \%$ ) o mais freqüente, por difusão nos tecidos circunjacentes. Observaram-se, também, 4 casos (26\%) de hipertropia por acometimento do músculo reto inferior pela substância, e ainda $1(6 \%)$ caso de hemorragia retrobulbar devido a falha na técnica da injeção. Os casos que apresentaram ptose palpebral regrediram em torno de 1 mês após aplicação, e a hipertropia entre o primeiro e segundo mês pós-aplicação.

Não foi observada qualquer complicação sistêmica.

\section{DISCUSSÃO}

Toda vez que ocorre paralisia de um músculo extraocular há uma tendência que seu antagonista sofra contratura, após curto período de tempo. A contratura de um músculo extraocular, conforme mostrou Scott ${ }^{12}$, é traduzida anatomicamente por redução do número de sarcômeros e clinicamente por restrição ao teste de ducção forçada. Vale lembrar que uma cirurgia de recuo (enfraquecimento) de um músculo 


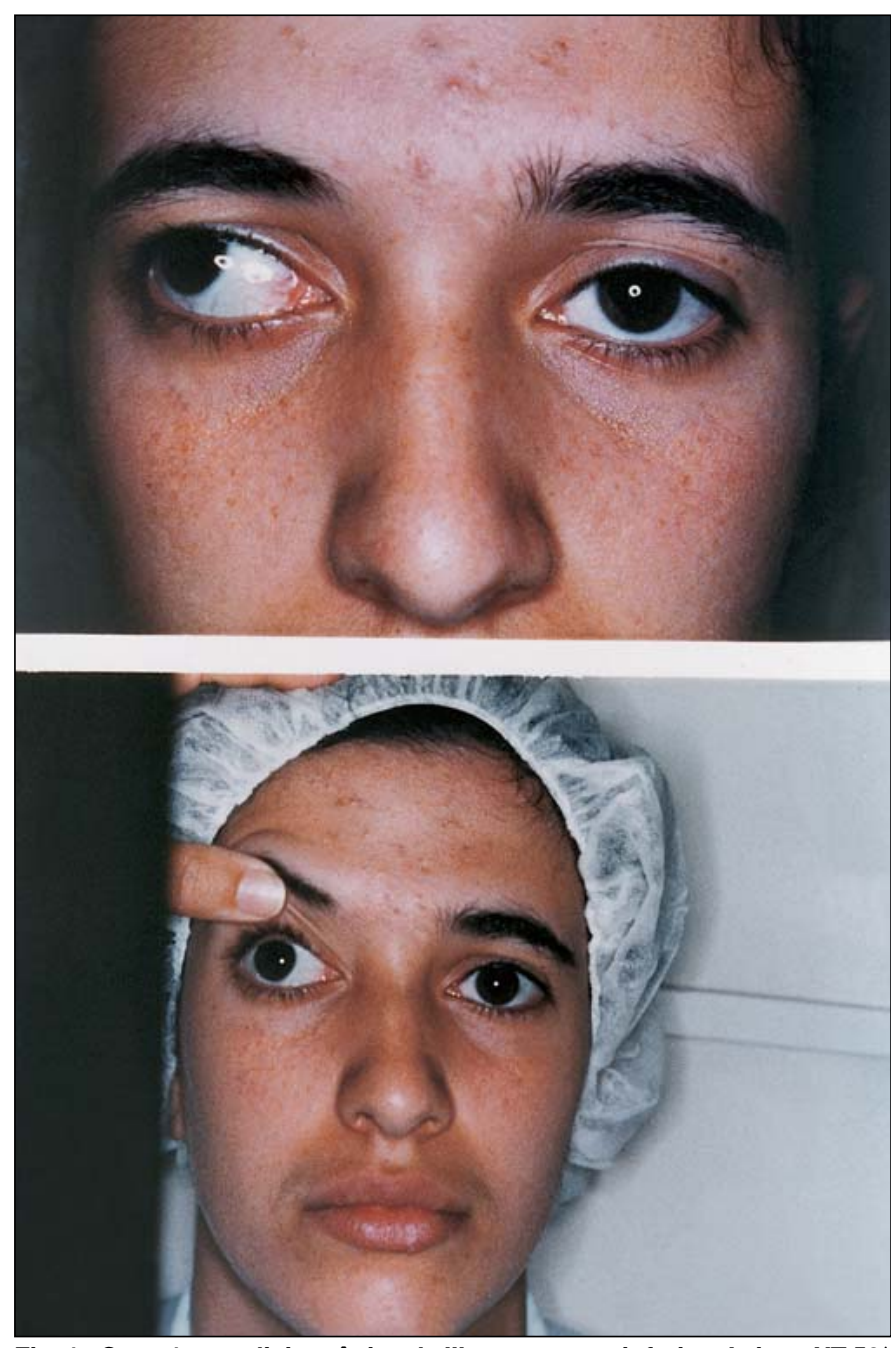

Fig. 1 - Caso 1 - paralisia crônica de III nervo, ramo inferior. Acima: XT 50 + HTD 20 (pré-injeção) Abaixo: XT 30 + HTD $10^{\Delta}$ (2 meses pós- injeção)

contraturado simplesmente modifica a posição deste músculo em relação ao globo ocular, mas não modifica sua contratura, o que clinicamente causa alterações nas duç̧ões oculares: por exemplo, um músculo reto medial recuado em excesso causa deficiência de adução; se este músculo for recuado menos que o necessário, também não elimina a restrição de abdução.

A toxina botulínica, por produzir paralisia temporária no músculo contraturado, possibilita que este volte ao seu comprimento normal e a ter o número de sarcômeros também normal ${ }^{12}$. Isto significa que, ao normalizar anatomicamente $o$ músculo submetido à aplicação da toxina botulínica, não há necessidade de cirurgia.

É obvio que em uma paralisia definitiva, como a ação da toxina botulínica é temporária, se nenhum procedimento cirúrgico adicional for acrescentado à aplicação, o músculo antagonista volta a contraturar-se e o olho tende a voltar ao desvio inicial. Por este motivo, é essencial indicar ou associar cirurgia de reforço ou transposições musculares dos
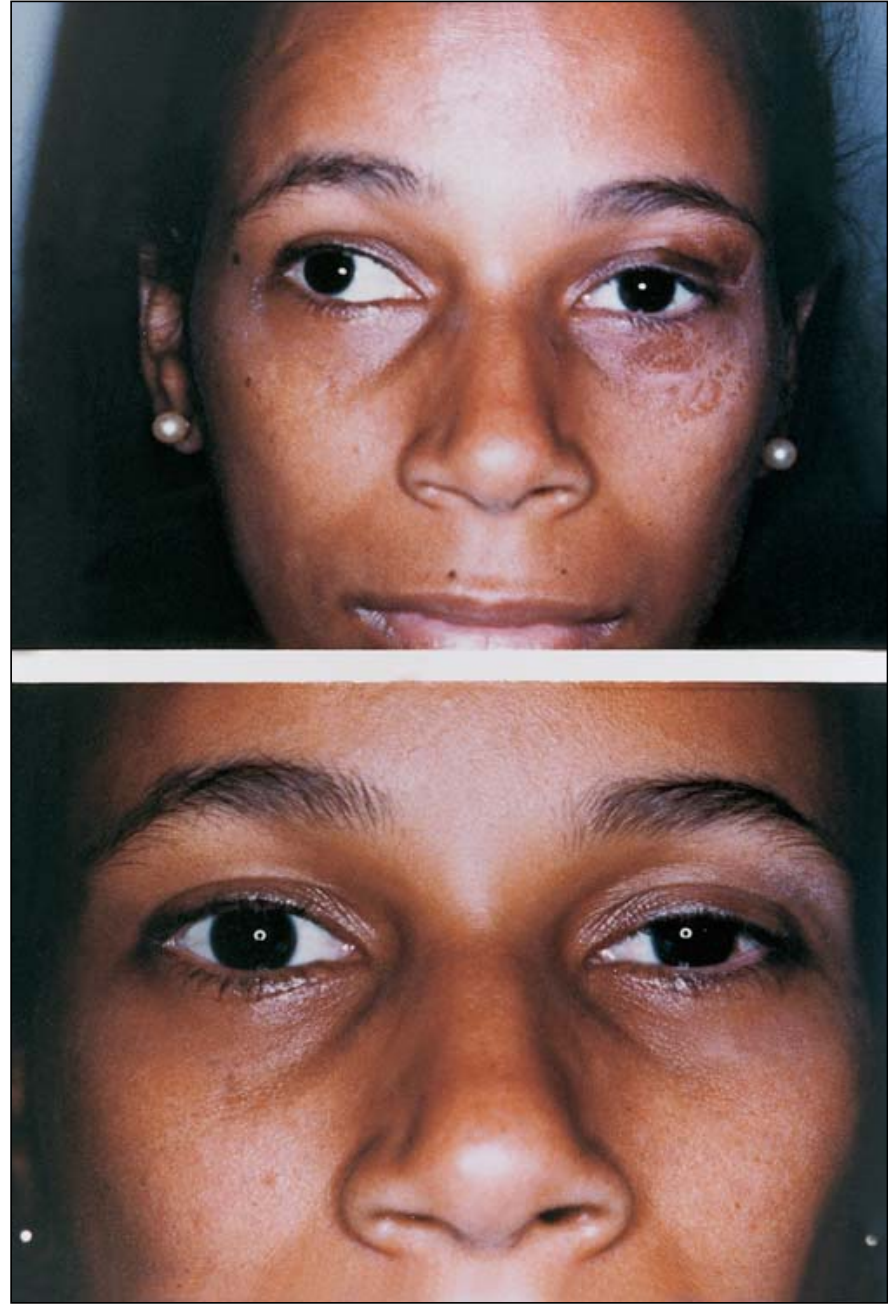

Fig. 2 - Caso 4 - paresia de III nervo, ramo inferior. Acima: XT 25 ${ }^{\Delta}$ HTD 10 (pré-injeção) Abaixo: X $8^{\Delta}$ (6 meses pós- injeção)

músculos paralisados, a fim de reequilibrar as forças ativas $\mathrm{e}$ passivas.

Nosso trabalho comprova que a injeção de toxina botulínica funciona como fator enfraquecedor e elimina a contratura do músculo aplicado ${ }^{4,6,13}$, como foi observado nos casos $4,6,10,12,13,14$ e 15 . Nestes casos não houve necessidade de cirurgia, pois o desvio diminuiu ou simplesmente se tratava de paresia muscular.

A indicação de tratamento em todos os casos foi funcional, pois todos eles apresentavam diplopia. Onze pacientes (73\%) obtiveram fusão em PPO, após o último procedimento realizado, o que consideramos um bom resultado por se tratar de uma série de pacientes de difícil tratamento.

Dos 11 pacientes que apresentaram paralisia do VI nervo, $4(36 \%)$ puderam controlar o desvio com o medicamento (seguimento de 4 meses), sendo todos esses pacientes casos de paralisias agudas. Nos outros 7 pacientes (63\%), associamos cirurgia a fim de obter resultado favorável que permitisse ao paciente obter fusão em PPO. 


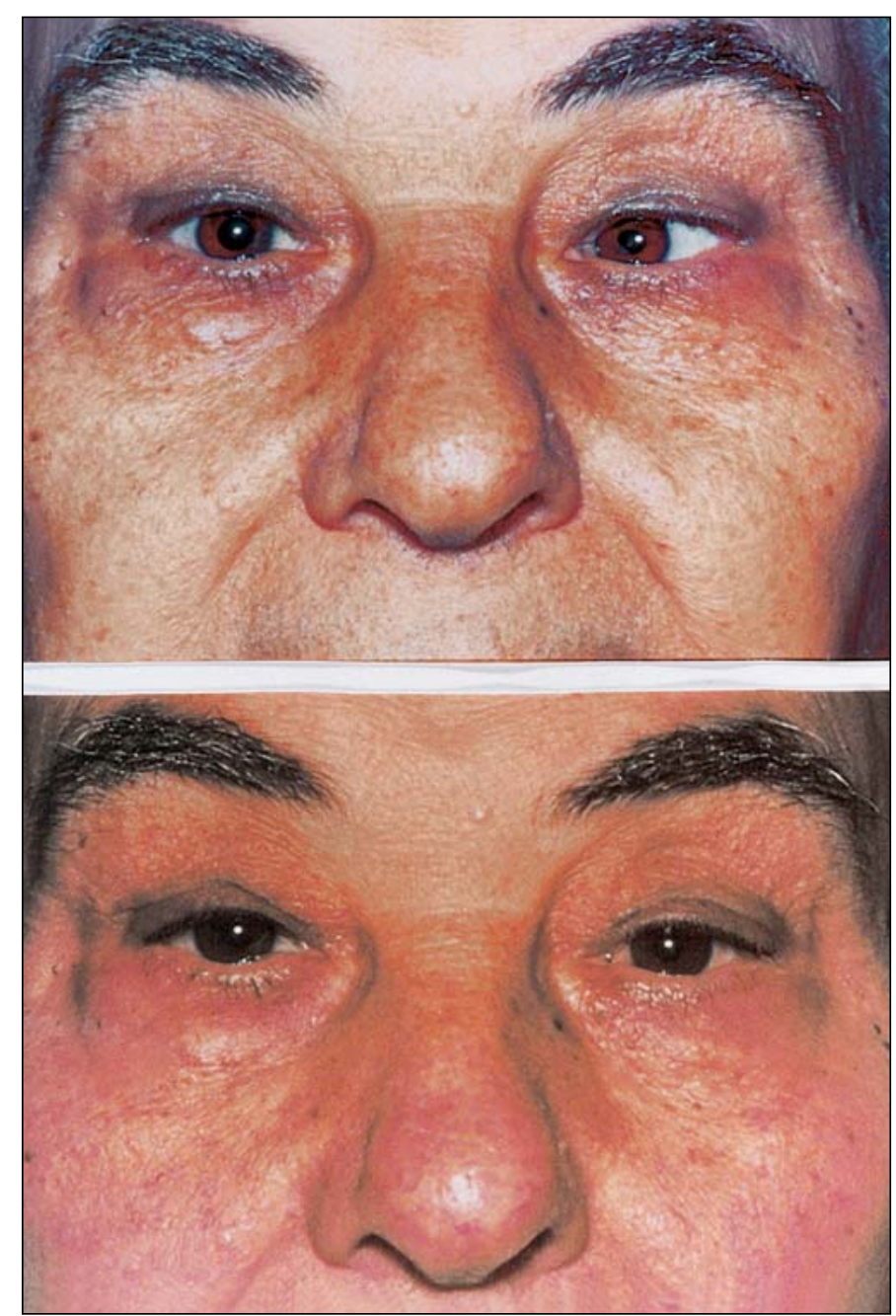

Fig. 3 - Caso 15 - Paralisia aguda de VI nervo. Acima: ET 30 (préinjeção) Abaixo: ORTO (4 meses pós- injeção)

Com isto, observamos que a toxina botulínica funciona como alternativa ao tradicional procedimento cirúrgico, nos casos agudos de VI nervo, e que, quando existe função ausente do músculo reto lateral, a toxina não será efetiva como único tratamento para controlar o desvio.

Dos 4 casos que apresentaram paralisia do III nervo, 3 pacientes $(75 \%)$ obtiveram ortotropia após a aplicação e só em 1 caso (25\%), houve necessidade de associar cirurgia.

A ausência de resposta em alguns de nossos casos após a primeira aplicação pode ter sido por falha técnica na aplicação ou devido à fibrose de tecidos adjacentes, e não por falta de resposta ao próprio medicamento.

Esta pesquisa permitiu-nos concluir que, nos casos em que havia força muscular residual no músculo afetado e bom potencial de fusão, a toxina botulínica foi o melhor tratamento, pois foi possível obter bom posicionamento ocular que permitisse fusão, sem associar cirurgia.

\section{SUMMARY}

Purpose: To evaluate the treatment by injection of botulinum toxin in acquired sixth and third nerve palsies.

Methods: Fifteen patients were treated with botulinum toxin. They were studied prospectively during nine months, between August, 1998 and May, 1999. In addition to the strabismus examination, a complete ophthalmological evaluation was performed. The patients were followed for 2 to 7 months after the last injection or surgical procedure. Results: Eleven patients (73\%) presented with sixth nerve palsy and four (27\%) with third nerve palsy. Six cases were acute (40\%) and 9 cases (60\%) were chronic. Five acute cases (83\%) obtained ocular alignment and controlled the deviation with toxin. Two of nine chronic cases (22\%) obtained orthotropia after the last injection. Seven chronic cases were treated with toxin and surgery; 4 of these (46\%) obtained acceptable ocular aligment and the remaining three cases (32\%) did not obtain ocular aligment with this treatment.

Conclusion: The toxin was the best treatment for the cases that had residual muscle function and good potential for fusion after sixth and third nerve palsies, because it controlled the deviation and then fusion was obtained without surgery.

Keywords: Botulinum toxin; Acquired paralytic strabismus.

\section{REFERÊNCIAS BIBLIOGRÁFICAS}

1. Bramowicz AM. (Ed.): Moricizine for Cardiac Arrhytmias. The Medical Letter 1990;32:99-105.

2. Scott AB, Rosenbaum A, Collins CC. Pharmacologic weakening of extraocular muscles. Invest Ophthalmol 1973;12:924-7.

3. Gammon JA Chemodenervation treatment of strabismus and blepharospasm with botulinum toxin. Ocular Therapy 1984;1:3-7.

4. Scott AB - Botulinum toxin injection of eye muscles to correct strabismus. Trans Am Ophthalmol Soc 1981;79:734-70.

5. Osako M, Keltner JL. Botulinum A toxin $\left(\right.$ Oculinum $\left.^{\circledR}\right)$ in Ophthalmology. Surv Ophthalmol 1991;36:28-46.

6. Biglan AW, Burnstine RA, Roger GL, Saunders RA. Management of Strabismus with Botulinum A toxin. Ophthalmology 1989;96:935-43.

7. Scott AB. Botulinum toxin injection into extraocular muscles as an alternative to strabismus surgery. Ophthalmology 1980;87(10):1044-9.

8. Scott AB. Botulinum Toxin Treatment of Strabismus. Focal Points: Clinical Modules for Ophthalmologists. San Francisco, American Academy of Ophthalmology 1989;7:1-11.

9. Sellin LC. The pharmacological mechanism of botulism. Trends in Pharmacological Science 1985;6:80-2.

10. Scott AB, Kraff S P. Botulinum Toxin Injection in the Management of Lateral Rectus Paresis. Ophthalmology 1985;92:676-83.

11. Mc Neer KW. Botulinum Toxin Management of Essential Infantile Esotropia in children. Arch Ophthalmol 1997;115:1411-8.

12. Scott AB. Change of eye muscle sarcomere according to eye position. J Pediatr Ophthalmol Strabismus 1994;31(2):85-8.

13. Metz HS, Snell L. Botulinum toxin chemodenervation in infants and children. J Pediatr Ophthalmol Strabismus 1984;21:110-3. 